\title{
Camera Calibration Using Multiple Images
}

\author{
Paul Beardsley, David Murray, Andrew Zisserman * \\ Robotics Group, Dept. of Engineering Science, University of Oxford, Oxford OX1 3PJ, UK. \\ tel: +44-865 273154 fax: +44-865 273908 email: [pab,dwm,az]@uk.ac.ox.robots
}

\begin{abstract}
This paper describes a method for camera calibration. The system consists of a static camera which takes a sequence of images of a calibration plane rotating around a fixed axis. There is no requirement for any exact positioning of the camera or calibration plane.

From each image of the sequence, the vanishing points and hence the vanishing line of the calibration plane are determined. As the calibration plane rotates, each vanishing point moves along a locus which is a conic section, and the vanishing line generates an envelope which is also a conic section. We describe how such conics can be used to determine the camera's focal length, the principal point (the intersection of the optic axis with the image plane), and the aspect ratio.
\end{abstract}

\section{Introduction}

The formation of images in a camera is described by the pinhole model. This model can be specified by the camera's focal length, principal point, and the aspect ratio. These parameters are called intrinsic because they refer to properties inherent to the camera. In contrast, extrinsic parameters describe the translation and rotation of a camera with respect to an external coordinate frame and are thus solely dependent on the camera's position, not on its inherent properties. The calibration method in this document measures intrinsic parameters only.

Camera calibration is a central topic in the field of photogrammetry [ $P h 80]$. In computer vision, the best-known calibration method is due to Tsai [Ts86] [LT88]. Other important references include [Ga84], [FT87], [Pu90]. Recently, calibration methods based on the use of vanishing points have been proposed [CT90], [Ka92]. The calibration method here uses vanishing points, the novel aspect of the work being that vanishing points are shown to move in a predictable way for constrained scene motions. This offers a way of integrating data over many images prior to calibrating the camera. The motivation for utilising more data is to obtain resilience to noise.

\section{Intrinsic Parameters}

Figure 1(a) shows the standard pinhole model of a camera. $O$ is the point through which all rays project and is referred to as the optic centre. The perpendicular from $O$ to the image plane intersects the image plane at the principal point, $P$. The line $O P$ is the optic axis and the distance $O P$ is the focal length. Figure 1(b) shows the customary way in which the physical system is actually represented, with a right-handed coordinate frame

\footnotetext{
* This work was supported by SERC Grant No GR/G30003. PB is in receipt of a SERC studentship. AZ is supported by SERC.
} 
$\hat{\mathbf{x}}_{\mathbf{c}} \hat{\mathbf{y}}_{\mathbf{c}} \hat{\mathbf{z}}_{\mathbf{c}}{ }^{2}$ having origin $O$ and $z$-axis aligned with $O P$. The ratio of the scaling along the $y$-axis to the scaling along the $x$-axis is called the aspect ratio.

(a)

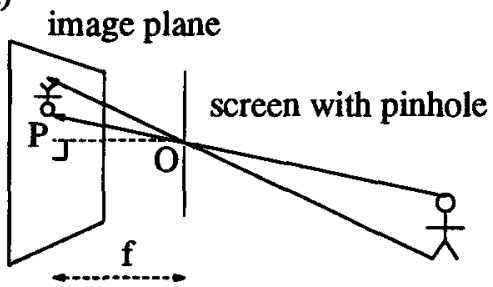

(b)



Fig. 1. The pinhole model. (a) shows the standard model for image formation - rays of light from the scene pass through the pinhole and strike the image plane creating an inverted image; (b) the usual diagrammatic representation of the system with a right-handed coordinate frame set up at $O$.

A real camera lens does not act like a perfect pinhole, so there are deviations from the model above. The most significant of these is known as radial distortion. The form of radial distortion and methods for its correction are described in [Ph80], [Be91].

\section{Vanishing Point and Vanishing Line Information}

\subsection{Vanishing Points and Vanishing Lines}

Semple [SK52] is a comprehensive reference for the properties of vanishing points and vanishing lines - this section outlines some of the elementary results. Figure 2 shows a camera viewing a plane. Parallel lines on the plane appear in the image as converging lines, which are concurrent at a vanishing point. The figure shows two sets of parallel lines on the plane with different orientations - the line through their vanishing points is called the vanishing line (the horizon) of the plane. Any set of parallel lines on the plane will have a vanishing point on this same vanishing line.

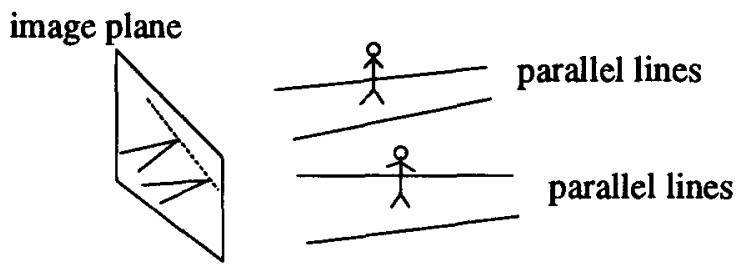

Fig. 2. The parallel lines in the scene appear as converging lines in the image. Each set of parallel lines has its own vanishing point in the image. The line through the vanishing points is the vanishing line (the horizon) of the plane in the scene.

Vanishing points and vanishing lines are important sources of information about the geometry of the scene. A vector at the optic centre pointing towards a vanishing point

\footnotetext{
${ }^{2} \mathbf{v}$ is used to denote a vector of arbitrary magnitude, and $\hat{v}$ to denote a unit vector.
} 
on the image plane is parallel to the physical lines in the scene which have given rise to the vanishing point. Also, a plane which passes through the optic centre and a vanishing line on the image plane has the same normal as the physical plane which has given rise to the vanishing line [Ka92].

\subsection{Generating Conics from Vanishing Points and Vanishing Lines}

The main idea in the proposed calibration method is that, given a static camera observing constrained motion of a calibration plane, vanishing points and vanishing lines move in a predictable way. Figure 3 illustrates the physical setup of the system. From an image of the calibration plane, it is possible to obtain a number of vanishing points and hence the vanishing line of the plane. This section shows that rotation of the calibration plane causes a vanishing point of the plane to move along a conic, and causes the vanishing line of the plane to generate an envelope (Fig. 7) which is a conic.

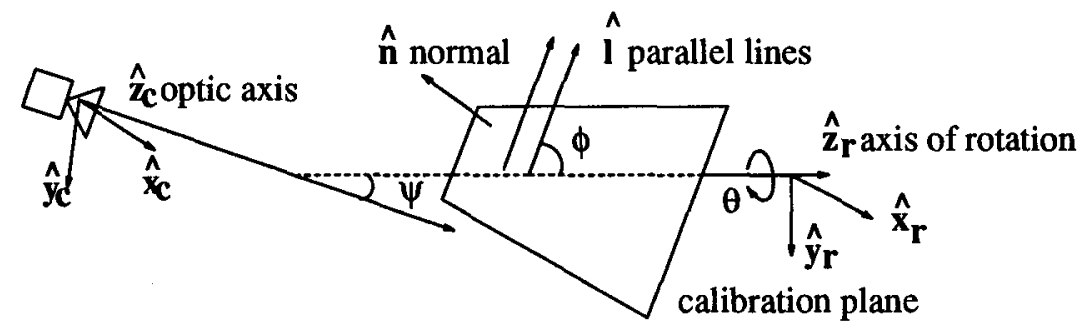

Fig. 3. The physical system. A camera is pointing at a calibration plane which is slanted away from the fronto-parallel position. The calibration plane is mounted on an axis $\mathbf{z} \mathbf{r}$ about which it can be rotated, and $\hat{\mathbf{z}}_{\mathbf{r}}$ is skewed away from the optic axis $\hat{\mathbf{z}}_{\mathbf{c}}$. There is no requirement for any exact positioning of the camera or calibration plane.

Referring to Fig. 3, consider a set of parallel lines on the calibration plane with direction given by the unit vector $\hat{1}$. As the plane rotates, the direction of $\hat{\mathbf{l}}$ changes in the way described by the following parametric representation (in spherical polar coordinates)

$$
\hat{\mathbf{l}}(\theta)=\sin \phi \cos \theta \hat{\mathbf{x}}_{\mathbf{r}}+\sin \phi \sin \theta \hat{\mathbf{y}}_{\mathbf{r}}+\cos \phi \hat{\mathbf{z}}_{\mathbf{r}}
$$

where $\hat{\mathbf{x}}_{\mathbf{r}} \hat{\mathbf{y}}_{\mathbf{r}} \hat{\mathbf{z}}_{\mathbf{r}}$ is an orthonormal set of vectors with $\hat{\mathbf{z}}_{\mathbf{r}}$ parallel to the axis of rotation, $\phi$ is a constant given by $\cos \phi=\hat{\mathbf{l}}$. $\hat{\mathbf{z}}_{\mathbf{r}}$, and $\theta$ is the angle of rotation with range $0 \leq \theta<2 \pi$.

It was pointed out in Sect. 3.1 that a vector passing through the optic centre and a vanishing point is parallel to the physical lines in the scene which have given rise to the vanishing point i.e. given a set of parallel lines $\hat{\mathbf{l}}\left(\theta_{0}\right)$ on the calibration plane, there is a parallel vector $\hat{\mathbf{I}}_{\mathbf{c}}\left(\theta_{0}\right)$ located at the optic centre and passing through the vanishing point for the lines. Now, the motion of the physical lines is described by (1), so the motion of the associated vector passing through the optic centre and the vanishing point is also described by (1). A vector moving in this way sweeps out a circular cone. It follows that the locus of the vanishing point is given by the intersection of a circular cone and the image plane, and therefore the locus of the vanishing point is a conic section. This is illustrated in Fig. 4. An analogous argument can be used to show that, as the calibration plane rotates, the vanishing lines generate an envelope which is a conic section.

A mathematical analysis is omitted, but the main results are summarised. 
1. Although the physical setup is actually capable of generating any type of conic, the only conic sections considered in the remainder of the paper are ellipses. It can be shown that the equation of the ellipse on the image plane is

$$
\frac{\left(\cos ^{2} \psi-\sin ^{2} \psi \tan ^{2} \phi\right)^{2}}{f^{2} \tan ^{2} \phi}\left[x-\frac{f \sin \psi \cos \psi\left(1+\tan ^{2} \phi\right)}{\cos ^{2} \psi-\sin ^{2} \psi \tan ^{2} \phi}\right]^{2}+\frac{y^{2}\left(\cos ^{2} \psi-\sin ^{2} \psi \tan ^{2} \phi\right)}{f^{2} \tan ^{2} \phi}=1
$$

where the origin of the coordinate frame is at the principal point, and the $x$-axis is aligned with the major axis of the ellipse; $\phi$ is the semi-angle of the cone and $\psi$ is the angle between the optic axis of the camera and the axis of rotation of the calibration plane (see Figs. 3 and 4 ); $f$ is the focal length.

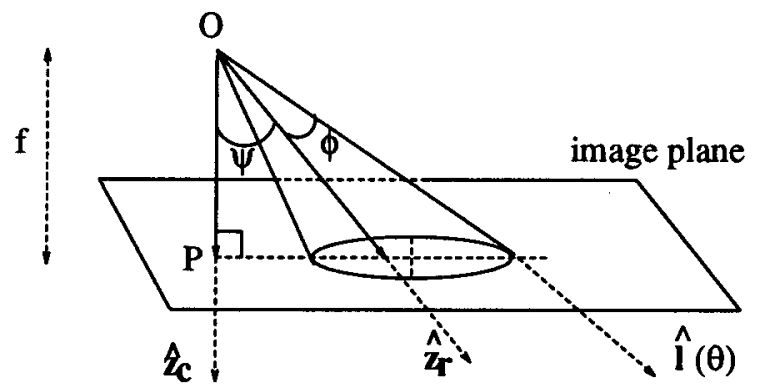

Fig. 4. This figure illustrates the locus of the vanishing point. The optic axis of the camera is $\hat{\mathbf{z}}_{\mathbf{c}}$. The axis of the circular cone is parallel to the axis of rotation of the calibration plane, $\hat{\mathbf{z}}_{\mathbf{r}}$. The circular cone is swept out by a vector parallel to the direction of the parallel lines on the calibration plane, $\hat{\mathrm{l}}(\theta)$. The vanishing point moves along the intersection of the cone and the image plane. The semi-angle of the cone is $\phi$, and the skew angle between the optic axis and the axis of the cone is $\psi$. Note that the axis of the cone does not pass through the centre of the ellipse.

2. For a particular configuration of the system, the vanishing points and vanishing lines generate ellipses with collinear major axes, although with different minor axes and eccentricities (depending on their associated $\phi$ value). The common major axis passes through the principal point.

3. As shown in Fig. 4, the direction of the major axis is determined by the optic axis and the axis of the cone (which is parallel to the axis of rotation of the calibration plane). This direction is fixed for a particular configuration of the system, but can be adjusted by moving the camera or the axis of rotation of the calibration plane.

4. The ellipses used in the actual experiments were generated from vanishing line envelopes rather than from vanishing points - a vanishing line is a best-fit line through a number of vanishing points and should therefore be more resilient to noise.

\section{Circular Cones and the Optic Centre}

This section considers what information an ellipse on the image plane provides about the position of the optic centre.

Figure 5 shows an ellipse on the image plane, which is either the locus of a vanishing point, or the envelope of a set of vanishing lines. The discussion in Sect. 3 assumed a 
known camera geometry and showed how vanishing point and vanishing line information can give rise to an ellipse. Camera calibration is the inverse process - given an ellipse generated from vanishing points or vanishing lines, determine the camera geometry.

Now the ellipse can be regarded as the intersection of the image plane with a circular cone whose vertex is at the optic centre. Thus, the problem is to use the ellipse to determine the set of all circular cones which could have given rise to it; more specifically, it is the vertices of this set of cones which are of interest since the optic centre must coincide with one of these vertices. It is shown below that the vertices lie on a hyperbola in a plane perpendicular to the image plane, as illustrated in Fig. 5. Thus, a single ellipse leaves the position of the optic centre underdetermined, since it can lie anywhere along the hyperbola. Given two distinct ellipses, the optic centre lies at the intersection of the two associated hyperbolae, and hence its position is uniquely determined (actually there are four points of intersection, pairwise symmetric on either side of the image plane - the incorrect solutions on the wrong side of the image plane are automatically eliminated, and the other incorrect solution is easily eliminated because it lies far from the centre of the image).

As shown in Fig. 5, a coordinate frame $\hat{\mathbf{x}}_{\mathbf{e}} \hat{\mathbf{y}}_{\mathbf{e}} \hat{\mathbf{z}}_{\mathbf{e}}$ is set up with the $\hat{\mathbf{x}}_{\mathbf{e}} \hat{\mathbf{y}}_{\mathbf{e}}$ plane coincident with the image plane, the $\hat{\mathbf{x}}_{\mathbf{e}}$ axis aligned with the major axis of the ellipse, and the origin at the centre of the ellipse.

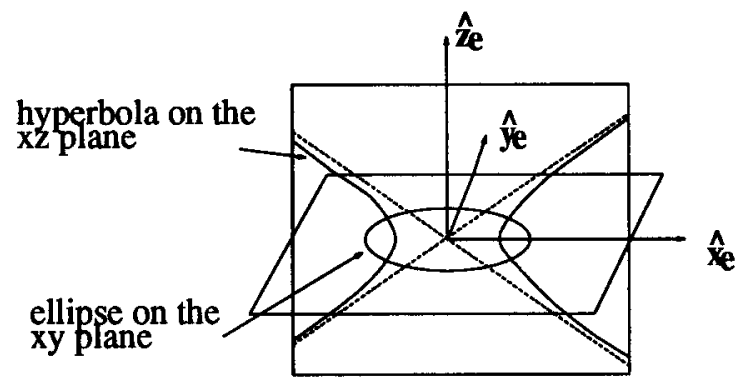

Fig. 5. Note that this is a $S D$ diagram, with the ellipse on the the $\hat{\mathbf{x}} \mathbf{e}$ ye plane (the image plane), and the hyperbola on the $\hat{\mathbf{x}}_{\mathrm{e}} \hat{\mathbf{z}}_{\mathrm{e}}$ plane. The set of circular cones which could give rise to the ellipse have their vertices on the hyperbola. As described in the text, possible positions of the optic centre lie on this same hyperbola.

Points $\mathbf{x}$ on the surface of a cone with vertex $\mathbf{v}$, axis $\hat{\mathbf{w}}$, and semi-angle $\phi$ are described by

$$
|\mathbf{x}-\mathbf{v}|^{2} \cos ^{2} \phi-(\hat{\mathbf{w}} \cdot(\mathbf{x}-\mathbf{v}))^{2}=0
$$

By expressing the intersection of the cone and the image plane in the canonical form for an ellipse, which is

$$
\frac{x^{2}}{a^{2}}+\frac{y^{2}}{b^{2}}=1
$$

it is possible to derive the following constraint on the cone vertices

$$
\frac{v_{x}^{2}}{a^{2}-b^{2}}-\frac{v_{z}^{2}}{b^{2}}=1
$$

Thus, the cone vertices $\mathbf{v}$ lie on a hyperbola in the $\hat{\mathbf{x}}_{\mathbf{e}} \hat{\mathbf{z}}_{\mathbf{e}}$ plane. When $v_{z}=0, v_{x}=$ $\sqrt{a^{2}-b^{2}}$. This is the focus of the ellipse, so the hyperbola goes through the ellipse foci.

Equation (4) will be used in the computation of focal length. 


\section{Camera Calibration}

\subsection{Determination of Aspect Ratio}

The determination of aspect ratio relies on the following observation - given a set of ellipses, all the major axes are concurrent at one point (the principal point). If the aspect ratio is not correct, however, the ellipses are distorted and the major axes will not be concurrent. Thus, adjusting the aspect ratio until the major axes are concurrent serves to identify the true aspect ratio. It is evident that this method requires a minimum of three ellipses with different major axes in order to carry out the concurrency test.

The experimental procedure is as follows. The system is set up so that the vanishing line envelope obtained from an image sequence will be an ellipse (the type of conic produced depends on the setting of the $\phi$ and $\psi$ angles defined in Sect. 3.2). An image sequence is taken as the calibration plane is rotated from 0 to $2 \pi$. The envelope of the vanishing lines measured during the sequence is used to determine an ellipse.

The system configuration is then adjusted so that the major axis of the vanishing line envelope lies in a different direction (the direction of the major axis is determined by the skew between the optic axis of the camera and the axis of rotation of the calibration plane as described in Sect. 3.2). A new image sequence is taken, and again the envelope of the vanishing lines is used to determine an ellipse.

This is repeated at least three times to produce the minimum of three ellipses required for the aspect ratio determination. Starting from an initial estimate, the aspect ratio is iteratively adjusted until the major axes of the ellipses are at their closest approach to concurrency - at this point, the value of the aspect ratio is recorded as the true aspect ratio. Concurrency is measured using a weighted least-squares test given in [Ka92].

We emphasise here that no accurate positioning is required when setting the camera and calibration plane using the guidelines above, and rough human estimation of position is always sufficient.

\subsection{Determination of Principal Point}

Once the aspect ratio has been corrected, all ellipses have major axes which are concurrent at the principal point. Thus, given two or more ellipses, it is possible to compute the principal point.

\subsection{Determination of Focal Length}

Once the principal point is known, the focal length can be found using a single ellipse. Focal length is computed using (4) from Sect. 4

$$
\frac{v_{x}^{2}}{a^{2}-b^{2}}-\frac{v_{z}^{2}}{b^{2}}=1
$$

$v_{x}$ is the offset of the centre of the ellipse from the principal point and is known; $a$ and $b$ are obtained directly from the ellipse; $v_{z}$ is the focal length and is the only unknown.

\section{Implementation and Results}

Six image sequences were taken, with varying system geometry as described in Sect. 5.1. The camera-calibration plane distance was about $80 \mathrm{~cm}$. There were 36 frames per image 


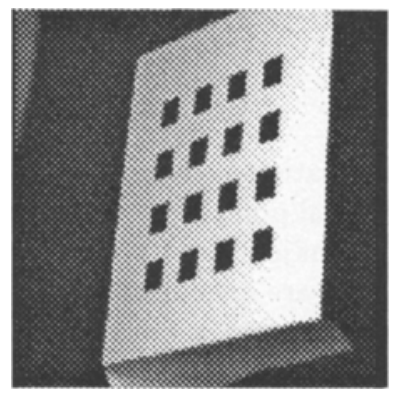

Fig. 6. Typical image of the calibration plane.

sequence and the calibration plane, which was mounted on a rotating table, was rotated about $10^{\circ}$ between each frame. A typical image is shown in Fig. 6.

Edges were found using a local implementation of the Canny edge detector which works to sub-pixel accuracy. The aspect ratio of the edge map was updated using a value which was close to the true aspect ratio, and the edgels were corrected for radial distortion using a priori knowledge of the radial distortion parameters $[\mathrm{Ph} 80],[\mathrm{Be} 91]{ }^{3}$ Best-fit lines were found for the 8 horizontal and 8 vertical lines available from the calibration grid, and 64 vertex positions were generated. Vanishing points were found in 16 different directions available from the vertices $\left(0^{\circ}, 90^{\circ}, \pm 45^{\circ}\right.$ etc. $)$, and the vanishing line was determined from the vanishing points.

At the end of the sequence, an ellipse was fitted to the envelope of the vanishing lines using the Bookstein algorithm $[B o 79]$ - the vanishing lines were represented in homogeneous coordinates and a line conic was determined. A point conic was then determined by inverting the matrix for the line conic [SK52].

Figure 7 shows the vanishing line envelopes for two example sequences. Figure 8 shows the major axes of ellipses fitted to the vanishing line envelopes of six sequences, after the aspect ratio has been corrected to bring them as close as possible to concurrency. The values determined for aspect ratio, principal point, and focal length are given in table 1 . The focal lengths were converted from pixels into millimetres using the pixel size provided in the camera specification.

The computed aspect ratio is in good agreement with the value obtainable from the camera and digitisation equipment specifications. The worst-case error for the perpendicular distances between the major axes and the computed principal point is about 12 pixels as shown in Fig. 8. The presence of this error is the subject of current investigation - Kanatani [Ka92] has pointed out that the Bookstein algorithm for conic fitting is inappropriate when there is anisotropic error in the data points. It is the case that there is anisotropic error in vanishing points and vanishing lines, and Kanatani's own algorithm for conic fitting is to be investigated. The standard deviation of the focal length indicates a $2 \%$ error - whether this is satisfactory or not depends on the particular application making use of the calibration information.

We are currently working on an error analysis. The calibration method is a linear process, so it should be possible to work through from the errors present in the Canny edge detection to an estimate of the variance in the output parameters. We hope that the analysis will show that the use of data from many frames causes cancelling of errors.

${ }^{3}$ Accurate radial distortion correction can be applied based on only approximate estimates of the aspect ratio and principal point. 

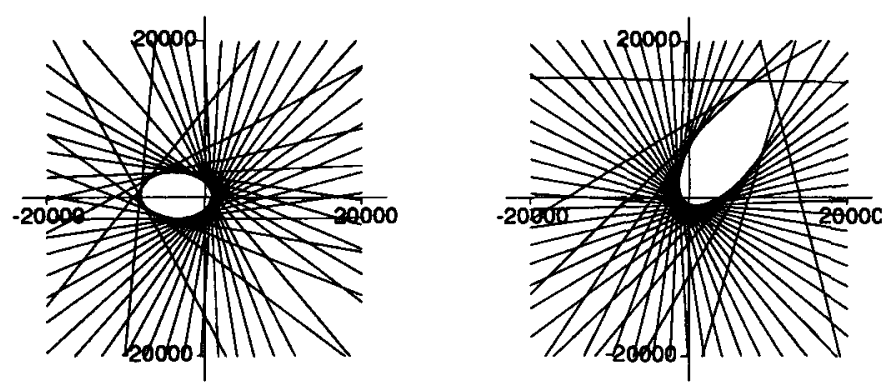

Fig. 7. This figure shows the envelope of vanishing lines for two example sequences. Axes are labelled with image plane coordinates in pixels. The aspect ratio has been corrected to an initial estimate of 1.51 .

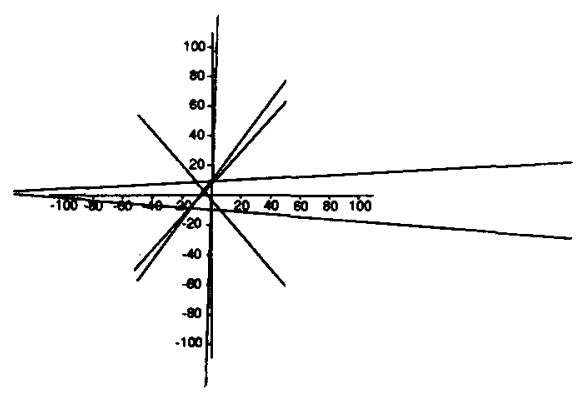

Fig. 8. This figure shows the closest approach to concurrency of the major axes of the ellipses, obtained by adjusting the aspect ratio. Axes are labelled with image plane coordinates in pixels. The least-squares estimation of the point of concurrency gives the principal point. The outliers are discussed in the text.

\section{Conclusion}

The most significant characteristic of the calibration method is that intrinsic parameters are computed in a sequential manner - first, the aspect ratio is found using a concurrency test which does not require knowledge of the other parameters; the principal point is then computed as the intersection point of a set of lines, a graphical computation which does not require the focal length; finally, the focal length is determined using the computed principal point together with the ellipse parameters. This breakdown of the processing gives rise to individual stages which are computationally simple and easy to implement, but it could cause problems through errors in one stage being passed on to subsequent stages.

The method makes use of image sequences, but the large amounts of data which this 


\begin{tabular}{|l|c|c|}
\hline Aspect ratio & 1.514 & \\
\hline Principal point $(x, y)$ & $(-4,1)$ & \\
\hline & Pixels & Millimetres \\
\hline Focal length sequence 1 & 1180 & 20.3 \\
Focal length sequence 2 & 1187 & 20.4 \\
Focal length sequence 3 & 1176 & 20.3 \\
Focal length sequence 4 & 1216 & 20.9 \\
Focal length sequence 5 & 1229 & 21.2 \\
Focal length sequence 6 & 1237 & 21.3 \\
\hline Focal length mean & 1204 & 20.7 \\
Focal length standard deviation & 26 & 0.4 \\
\hline
\end{tabular}

Table 1. Results for the measurement of aspect ratio, principal point and focal length.

involves are condensed to a single vanishing line from each image. The potential benefits of using many images are currently the subject of an error analysis.

\section{References}

[Be91] P.A. Beardsley et al. The correction of radial distortion in images. Technical report 1896/91. Department of Engineering Science, University of Oxford.

[Bo79] F.L. Bookstein Fitting conic sections to scattered data. Computer Graphics and Image Processing, pages 56-91, 1979.

[CT90] B. Caprile and V. Torre Using vanishing points for camera calibration. International Journal of Computer Vision, pages 127-140, 1990.

[FT87] O.D. Faugeras and G. Toscani The calibration problem for stereo. In Proc. of IEEE Conf Computer Vision and Pattern Recognition, Miami, 1987.

[Ga84] S. Ganapathy. Decomposition of transformation matrices for robot vision. In Proc. of IEEE Conference on Robotics, pages 130-139, 1984.

[Ka92] K. Kanatani. Geometric Computation for Machine Vision. Oxford University Press, Due for publication 1992/3.

[LT88] R.K. Lenz and R.Y. Tsai. Techniques for calibration of the scale factor and image center for high accuracy 3-D machine vision metrology. In IEEE Transactions Pattern Analysis and Machine Intelligence, pages 713-720, 1988.

[Ph80] Manual of Photogrammetry. American Society of Photogrammetry, 1980.

[Pu90] P. Puget and T. Skordas An optimal solution for mobile camera calibration. In Proc. First European Conf Computer Vision, pages 187-188, 1990.

[SK52] J.G. Semple and G.T. Kneebone. Algebraic Projective Geometry. Oxford University Press, 1952.

[Ts86] R.Y. Tsai. An efficient and accurate camera calibration technique for 3D machine vision. In Proc. of IEEE Conf Computer Vision, pages 364-374, 1986.

Thanks to Professor Mike Brady for advice, and to Professor Ken-ichi Kanatani for discussions on projective geometry. Thanks to Phil McLauchlan, Charlie Rothwell and Bill Triggs for invaluable help.

This article was processed using the $\mathrm{IAT}_{\mathrm{E}} \mathrm{X}$ macro package with ECCV92 style 